\title{
Rational design of a PKA-based sensor for cGMP
}

\author{
Robin Lorenz ${ }^{1 *}$, Daniel He $\mathrm{e}^{2,3}$, Choel Kim, ${ }^{4,5}$ Chinten J Lim², ${ }^{2,3}$ Friedrich W Herberg ${ }^{1}$ \\ From 7th International Conference on cGMP Generators, Effectors and Therapeutic Implications \\ Trier, Germany. 19-21 June 2015
}

\section{Background}

The cAMP-dependent protein kinase (PKA) and the cGMP-dependent protein kinase (PKG) are highly homologous enzymes differing in their specificity for cAMP and cGMP, respectively. Recent structure-function studies led to the identification of key residues responsible for cGMPspecificity in PKG [1]. Introduction of these amino acids into PKA switches its cyclic nucleotide selectivity [2]. Here we demonstrate that the same mutations turn a cAMPspecific FRET-based A-kinase activity reporter (AKAR) into a cGMP-specific reporter.

\section{Methods and results}

cGMP-specific threonine and arginine residues found in PKG I were introduced into both the $\mathrm{N}$-terminal and $\mathrm{C}$ terminal cyclic nucleotide binding domains (CNB-A and CNB-B) of the PKA regulatory subunit I $\alpha$ (RI $\alpha)$ using site-directed mutagenesis. The four resulting constructs were: wild type, CNB-A mutant (T192R/A212T), CNB-B mutant (G316R/A336T) and CNB-A/B mutant which has all aforementioned mutations.

While wild type RI $\alpha$ showed a strong selectivity for cAMP vs. cGMP in both in vitro binding and kinase activation studies, either CNB-A or -B mutants bound both nucleotides with equal affinity, resulting in PKA holoenzymes that were activated by both nucleotides equally. The CNB-A/B mutant had a significantly higher affinity for cGMP, and the corresponding PKA holoenzyme was activated selectively by cGMP.

Mouse embryonic fibroblasts derived from a PKA RI $\alpha$ knockout mouse were contransfected with the RI $\alpha$ constructs along with an AKAR to test their responsiveness for the cell-permeant analogs 8-CPT-cAMP and 8-CPTcGMP, respectively [3]. Our results showed that both the wild type and the CNB-B mutant do not respond to 8-CPT-cGMP. In contrast, the CNB-A mutant showed a

\footnotetext{
* Correspondence: robin.lorenz@uni-kassel.de

'Department of Biochemistry, University of Kassel, Kassel, Germany

Full list of author information is available at the end of the article
}

phasic response to 8-CPT-cGMP, and the response of the double mutant $\mathrm{CNB}-\mathrm{A} / \mathrm{B}$ sustained longer.

\section{Conclusion}

Cyclic nucleotide-dependent regulation of protein kinase activity is an important aspect of eukaryotic signal transduction. The vice versa specificity of PKA and PKG determines the fidelity of cAMP-PKA and cGMP-PKG pathways. We applied our understanding of cyclic nucleotide selectivity to cellular sensor design and showed that mutating four key residues within a cAMP-specific reporter switches it into a cGMP-specific reporter.

Cyclic nucleotide selectivity has apparently evolved through mutations in the CNB. The identification of these key residues will improve the design of cyclic nucleotide-selective cellular reporters.

\section{Authors' details}

'Department of Biochemistry, University of Kassel, Kassel, Germany. ${ }^{2}$ Department of Pediatrics, University of British Columbia, Vancouver, British Columbia, Canada. ${ }^{3}$ Child and Family Research Institute, BC Children's Hospital, Vancouver, British Columbia, Canada. ${ }^{4}$ Verna and Marrs McLean Department of Biochemistry and Molecular Biology, Baylor College of Medicine, Houston, TX 77030, USA. ${ }^{5}$ Department of Pharmacology, Baylor College of Medicine, Houston, TX 77030, USA.

Published: 2 September 2015

\section{References}

1. Huang GY, Kim JJ, Reger AS, Lorenz R, Moon EW, Zhao C, et al: Structural Basis for Cyclic-Nucleotide Selectivity and cGMP-Selective Activation of PKG I. Structure 2014, 22(1):116-124.

2. Lorenz R, Moon EW, Huang GY, Reger AS, Kim JJ, Franz E, Bertinetti D, Kim C, Herberg FW: Transforming PKA into PKG - a structure-function approach to understand cyclic nucleotide selectivity. BMC Pharmacology and Toxicology 2013, 14(Suppl 1):P41.

3. Allen MD, Zhang J: Subcellular dynamics of protein kinase A activity visualized by FRET-based reporters. Biochem Biophys Res Commun 2006, 348(2):716-721.

\section{doi:10.1186/2050-6511-16-S1-A64}

Cite this article as: Lorenz et al:: Rational design of a PKA-based sensor for cGMP. BMC Pharmacology and Toxicology 2015 16(Suppl 1):A64. 